\title{
NÃO-ADESÃO AO TRATAMENTO EM PACIENTES COM DOENÇA DE CROHN: prevalência e fatores de risco
}

\author{
Rita de Cássia Azevedo Couto CORNÉLI01, André Luis Tavares PINTO², \\ Fábio Heleno de Lima PACE ${ }^{3}$, Jussara Paixão MORAES ${ }^{4}$ e Júlio Maria Fonseca CHEBLI³
}

\begin{abstract}
RESUMO - Contexto - A não-adesão ao tratamento medicamentoso, em algum grau, ocorre universalmente. É uma das principais causas de insucesso no tratamento das doenças crônicas, tal como a doença de Crohn. Objetivo - Em doentes com doença de Crohn, avaliar a prevalência e os fatores de risco associados à baixa adesão ao tratamento medicamentoso. Métodos - No período entre julho de 2006 e julho de 2007 foram incluídos prospectivamente, para avaliação da não-adesão ao tratamento, 100 doentes com doença de Crohn em seguimento clínico no ambulatório de doenças inflamatórias intestinais. Os pacientes responderam ao Teste de Medida de Adesão a Tratamentos de Morisky e Green, modificado. De acordo com este teste, os pacientes foram classificados em dois grupos, conforme o grau de adesão: adesão e não-adesão. A não-adesão foi subdividida em intencional e não-intencional. Variáveis clínicas, psicológicas e farmacoterapêuticas foram pesquisadas na busca de possíveis fatores associados à não-adesão. Resultados - Entre os pacientes avaliados, $64 \%$ apresentaram escore compatível com não-adesão. O perfil mais frequente de não-adesão foi o do tipo não-intencional, e os pacientes mostraram ter conhecimento e motivação para o tratamento. Na comparação entre os dois grupos observou-se somente uma tendência a não-adesão entre os pacientes mais jovens $(P=0,07)$ e de raça não-branca $(P=0,06)$. Não houve correlação significativa entre o grau de adesão e as variáveis psicológicas e farmacoterapêuticas. Conclusões - Em pacientes com doença de Crohn, a prevalência de não-adesão ao tratamento medicamentoso é elevada (64\%). Indivíduos jovens e aqueles não-brancos parecem ser os mais predispostos à não-adesão. Portanto, é preciso estar alerta para sua ocorrência e, caso necessário, implementar medidas que busquem aumentar o grau de adesão destes pacientes.
\end{abstract}

DESCRITORES - Doença de Crohn. Recusa do paciente ao tratamento. Esquema de medicação.

\section{INTRODUÇÃO}

A doença de Crohn (DC) é uma afecção crônica de natureza sistêmica que pode afetar qualquer segmento do sistema digestório ${ }^{(22)}$. Tipicamente, sua história natural é marcada por períodos de atividade e remissão ${ }^{(34)}$. No mundo, diversos estudos têm demonstrado aumento significativo de sua incidência, principalmente na segunda metade do século $X X^{(10,31)}$. No Brasil, alguns estudos demonstraram tendência semelhante ${ }^{(11,35)}$. O tratamento da DC visa induzir ou manter sua remissão, evitar ou reduzir as complicações e melhorar a qualidade de vida dos pacientes afetados ${ }^{(11)}$. Na maioria dos pacientes existe necessidade de tratamento medicamentoso prolongado e, muitas vezes, por tempo indeterminado. $\mathrm{O}$ atual arsenal terapêutico inclui o uso de aminossalicilatos, antibióticos, glicocorticóides sistêmicos e não-sistêmicos, imunomoduladores e anticorpos monoclonais anti-fator de necrose tumoral (TNF) alfa ${ }^{(27)}$.
O comportamento de não-adesão ao tratamento medicamentoso é achado frequente em diversas doenças, sobretudo naquelas de natureza crônica e, provavelmente, constitui a causa mais importante de insucesso terapêutico ${ }^{(6)}$. Na DC, a complexidade de determinado tratamento que inclui regimes terapêuticos que necessitam a ingestão diária de vários comprimidos com diferentes horários de administração, seja durante a atividade ou a remissão da doença, pode influenciar negativamente o grau de adesão do paciente ${ }^{(18,37)}$. Também o acesso aos medicamentos utilizados no tratamento da DC pode ser dificultado ou mesmo impedido em função do alto custo destas drogas. Tal fato é apontado como preditor de nãoadesão ${ }^{(9)}$; entretanto, um estudo demonstrou que o fornecimento gratuito de medicamentos ao paciente não garante adesão adequada ${ }^{(8)}$. Além disso, fatores sociodemográficos, hábitos de vida e relacionamento com equipe de saúde podem influenciar no grau de

\footnotetext{
* Trabalho realizado no Núcleo de Pesquisa em Gastroenterologia, Ambulatório de Doenças Inflamatórias Intestinais, do Hospital Universitário da Universidade Federal de Juiz de Fora, MG.

${ }^{1}$ Hospital Universitário, Universidade Federal de Juiz de Fora - HU-UFJF, MG; ${ }^{2}$ Núcleo de Pesquisa em Gastroenterologia da UFJF; ${ }^{3}$ Disciplina de Gastroenterologia da Faculdade de Medicina, UFJF; ${ }^{4}$ Serviço de Gastroenterologia do HU-UFJF, Juiz de Fora, MG.

Correspondência: Dra. Rita de Cássia A. Couto Cornélio - Rua Catulo Breviglieri, 485/502 - Bairro Santa Catarina - 36036-110 - Juiz de Fora, MG.

E-mail: ritacornelio@acessa.com
} 
adesão terapêutica de determinado indivíduo ${ }^{(23)}$. Portanto, o grau de adesão a determinado tratamento tem natureza multifatorial.

Em um estudo que analisou o grau de adesão ao tratamento em doentes com doenças inflamatórias intestinais (DII), verificou-se não-adesão em $20 \%$ dos pacientes em tratamento de curta duração e em 50\% daqueles submetidos a terapias mais longas ${ }^{(15,21)}$. Tais níveis são similares àqueles observados em outras doenças crônicas ${ }^{(16)}$. No Brasil, em estudo recente realizado em um Hospital Universitário de Ribeirão Preto, $\mathrm{SP}^{(8)}$, lidando com pacientes com DII, foi observado, quando utilizada a análise de medicamentos (questionário padronizado), elevado grau de adesão, tanto na avaliação de 26 pacientes com DC (85\%), quanto para o mesmo número de pacientes com colite ulcerativa $(87 \%)$. Todavia, a aplicação do método de Morisky mostrou que $50 \%$ dos pacientes com DC e $63 \%$ daqueles com colite ulcerativa, apresentavam comportamento de baixa adesão.

A identificação de pacientes com DC que não aderem adequadamente ao tratamento proposto, pode permitir direcionar diversas intervenções multiprofissionais e estratégias que visem reforçar o comportamento de adesão, buscando não apenas o controle mais adequado de sua doença intestinal, mas também a melhoria da qualidade de vida desses pacientes ${ }^{(24)}$.

Apesar do crescente interesse por este tema com relação às DII, ainda existem poucos estudos publicados em nosso meio ${ }^{(7,8)}$ que descrevam índices e fatores de risco de não-adesão entre pacientes com DC. Além disso, os estudos disponíveis lidaram com casuística relativamente pequena e utilizaram população heterogênea de indivíduos (pacientes com DC e colite ulcerativa). Assim, depreende-se a importância da realização de estudos nacionais avaliando o comportamento de não-adesão em população maior e mais homogênea de pacientes com DII.

Neste estudo, objetivou-se avaliar em pacientes com DC a prevalência de não-adesão ao tratamento medicamentoso, bem como os possíveis fatores associados à mesma.

\section{MÉTODO}

\section{Desenho do estudo}

Estudo observacional e transversal realizado no ambulatório de Gastroenterologia Clínica - Centro de Doenças Inflamatórias Intestinais do Hospital Universitário da Universidade Federal de Juiz de Fora (UFJF), Juiz de Fora, MG. O Centro de DII é referência regional de atendimento especializado, abrangendo uma região com população estimada de 1.500 .000 habitantes. Atualmente, cerca de 460 doentes com DC estão em seguimento clínico neste setor.

\section{População de pacientes}

Entre julho de 2006 e julho de 2007 foram incluídos consecutivamente, 100 pacientes com DC em seguimento regular no Centro de DII, com idade entre 18 e 65 anos e que estavam em uso de pelo menos um medicamento. $\mathrm{O}$ diagnóstico de DC foi estabelecido pela combinação de dados clínicos e critérios endoscópicos ou radiológicos, suplementados com a aparência histológica típica em biopsias de mucosas ou espécimes de ressecção cirúrgica, quando disponíveis ${ }^{(19}$, ${ }^{20)}$. Foram excluídos pacientes com indicação de internação hospitalar ou de cirurgia imediata, decorrente de complicações da DII, aqueles com doença fulminante, presença de estoma ou de síndrome do intestino curto. Também não foi permitida a inclusão de as pacientes grávidas ou em fase de amamentação.

\section{Avaliação das características relacionadas aos doentes com DC}

Os seguintes dados foram registrados na inclusão do estudo: dados sociodemográficos, características clínicas relacionadas à DC (localização, fenótipo, atividade da doença, história cirúrgica prévia), tempo de acompanhamento no ambulatório, comorbidades, história atual de etilismo e tabagismo e informações farmacoterapêuticas. A avaliação da atividade, fenótipo e localização da DC foi realizada por um dos médicos do Serviço, não conhecedor dos dados relacionados à adesão dos pacientes. A localização e o fenótipo dos pacientes com DC foi descrito utilizando-se a classificação de Viena ${ }^{(12)}$. Para avaliação da atividade clínica da DC, utilizou-se o índice de sua atividade ${ }^{(2)}$, registrado com base nas informações do paciente, sobre seu estado geral na semana que antecedeu a consulta agendada. Considerou-se como doença em remissão, o escore menor que 150 pontos e doença em atividade, o escore acima de 150 pontos $^{(2)}$. As questões relativas à medicação e à doença, que por ventura não foram respondidas pelo paciente, foram esclarecidas através de consulta ao prontuário médico.

\section{Avaliação do grau de adesão ao tratamento}

O grau de adesão foi verificado por entrevista realizada pelo pesquisador, em local reservado, por uma única vez e de caráter individual, antes da consulta médica e mediante a aplicação de questionário estruturado denominado Teste de Medida de Adesão a Tratamentos (MAT) proposta por Morisky e Green e modificado por Sewicht ${ }^{(4,28,32)}$. A adesão foi avaliada considerando-se todos os medicamentos prescritos para o paciente, inclusive aqueles indicados para o tratamento de outras doenças concomitantes, eventualmente presentes.

O MAT modificado é um questionário baseado em quatro itens (Figura 1). A pontuação obtida varia de 0 a 4, atribuindo-se o valor 1 para cada resposta negativa obtida. Considerou-se alta adesão ao tratamento os pacientes que obtiveram escore de 4 pontos, média adesão escore de 2 a 3 pontos e baixa aquela com 0 a 1 ponto. As quatro perguntas iniciais do MAT modificado permitem classificar o comportamento do paciente com predisposição para não-adesão em dois tipos. Os que responderam sim às questões 1 e/ou 2 foram caracterizados como tendo comportamento de não-adesão do tipo não-intencional e aqueles que responderam sim às questões $3 \mathrm{e} / \mathrm{ou} 4$ como do tipo intencional ${ }^{(32)}$. Aos pacientes que responderam sim a pelo menos uma dessas questões iniciais, foram feitas duas perguntas adicionais com o objetivo de se determinar o motivo associado ao comportamento de não-adesão. Os que 


\begin{tabular}{|c|c|c|c|}
\hline \multicolumn{2}{|l|}{ ITENS } & \multicolumn{2}{|c|}{ PONTUAÇÃO } \\
\hline \multicolumn{2}{|c|}{$\begin{array}{l}\text { 1. Alguma vez você se esqueceu de tomar os medicamentos para } \\
\text { sua doença? }\end{array}$} & $\operatorname{Sim}(0)$ & Não (1) \\
\hline \multicolumn{2}{|c|}{$\begin{array}{l}\text { 2. Alguma vez você foi descuidado com os horários de tomar os } \\
\text { medicamento? }\end{array}$} & $\operatorname{Sim}(0)$ & Não (1) \\
\hline \multicolumn{2}{|c|}{$\begin{array}{l}\text { 3. Alguma vez você deixou de tomar os medicamentos para a sua } \\
\text { doença, por se sentir melhor? }\end{array}$} & $\operatorname{Sim}(0)$ & Não (1) \\
\hline \multicolumn{2}{|c|}{$\begin{array}{l}\text { 4. Alguma vez você deixou de tomar os medicamentos para a sua } \\
\text { doença, por iniciativa própria, após ter se sentido pior? }\end{array}$} & $\operatorname{Sim}(0)$ & Não (1) \\
\hline \multicolumn{4}{|c|}{ Na ocorrência de pelo menos uma resposta sim, aplicar as duas questões seguintes. } \\
\hline & Motivação & \multicolumn{2}{|c|}{ Conhecimento } \\
\hline $\begin{array}{l}\text { 5. Você foi informado sobre a importância e } \\
\text { benefício de usar o medicamento? }\end{array}$ & - & \multicolumn{2}{|c|}{$\operatorname{Sim} /$ não } \\
\hline $\begin{array}{l}\text { 6. Você se esquece de repor os medicamentos } \\
\text { antes que terminem? }\end{array}$ & $\operatorname{Sim} /$ não & \multicolumn{2}{|c|}{-} \\
\hline
\end{tabular}

responderam não à questão 5 foram considerados como não tendo conhecimento sobre a importância do tratamento e se a resposta foi sim na questão 6 , falta de motivação foi considerada como o motivo para a não-adesão ${ }^{(36,37)}$.

\section{Avaliação do estado psicológico}

Durante a entrevista para verificação do grau de adesão, os pacientes foram também avaliados quanto ao estado psicológico, utilizando-se instrumentos de auto-avaliação. Assim, o Inventário de Depressão Beck (BDI) ${ }^{(1)}$ e a subescala de ansiedade da Escala Hospitalar de Ansiedade e Depressão (AS-HADS) ${ }^{(38)}$, foram aplicados com o objetivo de se identificar a presença de sintomas depressivos e/ou ansiosos, respectivamente. O BDI é uma escala de 21 itens, com escores de resposta variando de 0 a 3 , que avalia a presença e gravidade de sintomas depressivos e a AS- HADS apresenta 7 itens que identificam a presença de ansiedade. Ambas já foram validadas em nosso meio ${ }^{(3,13)}$. No presente estudo, não se classificou a intensidade dos sintomas depressivos e/ou ansiosos, mas sim a sua presença (informação qualitativa). De acordo com a validação brasileira de ambas as escalas, pacientes com pontuação $\geq 15$ no BDI e pontuação $\geq 8$ na escala AS-HADS foram considerados com depressão e com ansiedade, respectivamente ${ }^{(3,13)}$.

\section{Análise estatística}

Para análise dos resultados, os pacientes foram divididos em dois grupos: adesão e não-adesão ao tratamento medicamentoso. Foram considerados como apresentando adesão aqueles que apresentaram 4 pontos na Escala de Morisky modificada, e não-adesão aqueles com 3 ou menos pontos na mesma escala.

A análise estatística foi realizada com a utilização do programa SPSS 14.0.0 (SPSS, Chicago, IL, EUA). As variáveis quantitativas foram expressas como mediana e variações ou, como média \pm DP, quando normalmente distribuídas. Foram calculadas estatísticas descritivas de todas as variáveis relevantes. A relação entre as variáveis e as possíveis associações com não-adesão foi verificada através do teste $t$ de Student ou do teste do qui ao quadrado. Foram consideradas associações estatisticamente significantes o valor de $P<0,05$.

\section{Considerações éticas}

O protocolo deste estudo foi aprovado pelo Comitê de Ética em Pesquisas da UFJF e todos os pacientes incluídos no estudo assinaram o termo de consentimento pós-informado.

\section{RESULTADOS}

\section{Pacientes}

Neste estudo, foram incluídos 100 pacientes, sendo 58 do sexo feminino e 86 da raça branca. A média de idade foi de $37,4 \pm 12,1$ anos. As características demográficas e clínicas dos pacientes estão demonstradas na Tabela 1 .

TABELA 1. Características demográficas e clínicas dos pacientes com doença de Crohn $(\mathrm{n}=100)$

\begin{tabular}{lc}
\hline Sexo & 58 \\
Feminino (n) & 42 \\
Masculino (n) & $37,4 \pm 12,1$ \\
Idade (anos)* & \\
Raça & 86 \\
$\quad$ Branca (n) & 14 \\
$\quad$ Não-branca (n) & 14 \\
Tabagismo & 7 \\
Consumo atual de álcool (n) & $39,7 \pm 36,8$ \\
Tempo de acompanhamento em ambulatório (meses)* & $63 \pm 56,6$ \\
Tempo de doença (meses)* & \\
Localização da doença & 48 \\
$\quad$ Íleo terminal (n) & 18 \\
Cólon (n) & 34 \\
Íleo-colônica (n) & \\
Fenótipo da doença & 48 \\
$\quad$ Não-estenosante, não-penetrante (n) & 21 \\
Estenosante (n) & 31 \\
Penetrante (n) & \\
Índice de atividade da doença & 31 \\
Remissão (n) & 39 \\
Atividade (n) & 31 \\
Presença de comorbidades (n) & \\
Cirurgia prévia (n) & 35 \\
\hline
\end{tabular}

Quanto às características clínicas da DC, o tempo médio de doença foi 63 meses e 69 pacientes encontravam-se em fase de remissão. O tempo médio de seguimento em ambulatório foi de 39,7 meses.

\section{Dados farmacoterapêuticos}

Foi frequente $(55 \%)$ o uso de medicamentos para outras comorbidades além dos prescritos para DC. O número de classes terapêuticas prescritas por paciente variou de um a nove. Em média, os pacientes incluídos no estudo tomavam cinco comprimidos por dia, distribuídos em duas administrações diárias.

A azatioprina e a prednisona foram os medicamentos mais prescritos para o tratamento da DC. Houve predomínio do 
fornecimento gratuito de medicamentos através do Sistema Único de Saúde.

\section{Prevalência de não-adesão}

A análise da adesão detectou a ocorrência de baixa em $16 \%$, média em $48 \%$ e alta em $36 \%$ dos pacientes. Desta forma, $64 \%$ dos pacientes foram considerados como apresentando não-adesão ao tratamento medicamentoso.

Os dados sobre não-adesão estão demonstrados na Tabela 2. O comportamento de não-adesão do tipo não-intencional $(58 \%)$ foi o predominante. Interessante observar que, apesar da elevada taxa de não-adesão, os pacientes mostraram ter conhecimento e motivação para adesão ao tratamento.

TABELA 2. Dados sobre não-adesão segundo o teste de Morisky modificado* $(\mathrm{n}=64)$

\begin{tabular}{lc}
\hline Tipo & $\mathbf{n}(\%)$ \\
\hline Não-intencional & $37(57,8)$ \\
Intencional & $2(3,1)$ \\
Ambos & $25(39,1)$ \\
Razão & \\
$\quad$ Falta apenas de conhecimento & $9(14,1)$ \\
$\quad$ Falta apenas de motivação & $14(21,9)$ \\
$\quad$ Falta de conhecimento e motivação & $4(6,2)$ \\
Falta de conhecimento ou motivação & $27(42,2)$ \\
\hline
\end{tabular}

*Adaptado a partir das referência SEWITCH et al. ${ }^{(32)}$ e CMAG-1 1

\section{Fatores de risco de não-adesão}

A Tabela 3 apresenta a relação entre o grau adesão ao tratamento e as características sociodemográficas e clínicas dos pacientes estudados. $\mathrm{Na}$ análise comparativa entre os dois grupos, não foi possível demonstrar nenhum tipo de associação estatisticamente significante com ocorrência de não-adesão (Tabela 3). No entanto, observou-se uma tendência de pacientes da raça não-branca $(P=0,06)$ e aqueles mais jovens $(P=0,07)$ apresentarem comportamento de nãoadesão. A presença de ansiedade ou depressão, assim como as variáveis farmacoterapêuticas não se correlacionou com o grau de adesão (Tabela 4).

\section{DISCUSSÃO}

O presente estudo demonstra elevada prevalência (64\%) do comportamento de não-adesão ao tratamento medicamentoso entre pacientes com DC. Esta taxa é algo superior àquela observada em outros estudos recentes $(40 \%$ a $50 \%$ ), que também avaliaram pacientes com $\mathrm{DC}^{(5,8)}$. Talvez, a definição mais ampla de não-adesão (que incluiu pacientes com baixa ou média adesão) utilizada no presente estudo, possa justificar, pelo menos parcialmente, este achado.

$\mathrm{Na}$ literatura atual, ainda não há um método considerado padrão-ouro para investigação da adesão ao tratamento medicamentoso. Nesta casuística, após a análise dos métodos disponíveis na literatura, optou-se pelo método indireto, utilizando-se o teste de Morisky com as modificações $\operatorname{propostas}^{(4,28,32)}$, que foi validado em estudos anteriores ${ }^{(4,7,}$ 16, 32, 36). Embora este método possa apresentar limitações ${ }^{(14)}$,
TABELA 3. Relação entre o grau de adesão ao tratamento e as características sociodemográficas e clínicas $(\mathrm{n}=100)$

\begin{tabular}{|c|c|c|c|}
\hline Variáveis & Adesão $(\mathrm{n}=36)$ & Não-adesão $(n=64)$ & $P$ valor \\
\hline \multicolumn{4}{|l|}{ Sexo } \\
\hline Feminino/masculino $\mathrm{n}(\%)$ & $24(66,7) / 12(33,3)$ & $34(53,1) / 30(46,9)$ & 0,18 \\
\hline Idade (anos)* & $40,2 \pm 11,7$ & $35,8 \pm 12,1$ & 0,07 \\
\hline \multicolumn{4}{|l|}{ Raça } \\
\hline Branca/não-branca (\%) & $34(94,4) / 2(5,6)$ & $52(81,3) / 12(18,8)$ & 0,06 \\
\hline Estado civil & & & 0,94 \\
\hline Casado n (\%) & $20(55,6)$ & $36(56,3)$ & \\
\hline Solteiro/separado n (\%) & $16(44,4)$ & $28(43,8)$ & \\
\hline Condição profissional & & & 0,47 \\
\hline Empregado/desempregado n (\%) & $24(66,7) / 12(33,3)$ & $38(59,4) / 26(40,6)$ & \\
\hline Escolaridade & & & 0,36 \\
\hline $1^{\circ}$ grau n $(\%)$ & $18(50,0)$ & $26(40,6)$ & \\
\hline $2^{\circ} / 3^{\circ}$ grau $\mathrm{n}(\%)$ & $18(50,0)$ & $38(59,4)$ & \\
\hline Tabagismo n (\%) & $4(11,1)$ & $10(15,6)$ & 0,53 \\
\hline Consumo atual de álcool n (\%) & $3(8,3)$ & $4(6,3)$ & 0,69 \\
\hline Tempo de doença (meses)* & $57,8 \pm 51,6$ & $65,9 \pm 59,5$ & 0,49 \\
\hline Índice de atividade da doença n (\%) & & & 0,94 \\
\hline Remissão n (\%) & $25(69,4)$ & $44(68,8)$ & \\
\hline Atividade n (\%) & $11(30,6)$ & $20(31,3)$ & \\
\hline Cirurgia prévia n (\%) & $16(44,4)$ & $19(29,7)$ & 0,13 \\
\hline $\begin{array}{l}\text { Tempo de acompanhamento } \\
\text { (meses)* }\end{array}$ & $40,1 \pm 36,9$ & $39,5 \pm 36,9$ & 0,90 \\
\hline Presença de comorbidades n (\%) & $10(27,8)$ & $21(32,8)$ & 0,60 \\
\hline
\end{tabular}

TABELA 4. Relação entre dados psicológicos, farmacoterapêuticos e grau de adesão $(n=100)$

\begin{tabular}{lccc}
\hline Variáveis & $\begin{array}{c}\text { Adesão } \\
(\mathbf{n}=36)^{*}\end{array}$ & $\begin{array}{c}\text { Não-adesão } \\
(\mathbf{n}=64)^{*}\end{array}$ & $\boldsymbol{P}$-valor \\
\hline Número de medicamentos* & $2,7 \pm 1,8$ & $2,4 \pm 1,3$ & 0,39 \\
Total de comprimidos/dia* & $5,2 \pm 3,1$ & $5,8 \pm 3,5$ & 0,44 \\
Número de administrações diárias* & $1,9 \pm 1,0$ & $2,2 \pm 1,2$ & 0,38 \\
Estado psicológico n(\%) & $10(27,8)$ & $25(39,1)$ & 0,25 \\
$\quad$ Depressão n(\%) & $15(41,7)$ & $30(46,9)$ & 0,61 \\
$\quad$ Ansiedade & $9(25)$ & $19(29,7)$ & 0,61 \\
$\quad$ Depressão e ansiedade & & & \\
Acesso ao medicamento & $22(61,1)$ & $38(59,4)$ & \\
$\quad$ Gratuitamente n(\%) & $4(11,1)$ & $3(4,7)$ & \\
$\quad$ Adquirido pelo paciente n(\%) & $10(27,8)$ & $23(35,9)$ & \\
$\quad$ Ambos n (\%) & & & \\
\hline * Média \pm desvio-padrão & &
\end{tabular}

os métodos diretos são pouco factíveis e também vulneráveis. Tais testes são baseados em técnicas analíticas que verificam se o medicamento foi administrado na dose e frequência necessárias, através da identificação de metabólitos dos medicamentos prescritos ou de marcadores químicos de maior permanência no organismo ${ }^{(26)}$. Entretanto, a utilização de marcadores bioquímicos (métodos diretos) tem baixa praticidade, é indisponível para a maioria das drogas e sua interpretação como medida de adesão é bastante complexa, em função das características individuais frente à metabolização de um fármaco ${ }^{(28)}$.

Alguns autores destacam que a entrevista poderia superestimar o grau de adesão, por ser este um comportamento esperado ${ }^{(14,}$ ${ }^{21}$. Contudo, no teste de Morisky a construção das questões 
pela negativa, em que a resposta "não" significa adesão, permite evitar os viéses de alguma propensão para a aquiescência ${ }^{(28)}$. $\mathrm{Na}$ presente série, onde a proposta foi detectar a tendência para a não-adesão ao tratamento, consideraram-se aderentes somente os pacientes que responderam negativamente a todas as questões do teste de Morisky. A resposta afirmativa a pelo menos uma das questões do teste, foi considerada como instrumento preditor de não-adesão. Dificilmente um paciente que declara não seguir o tratamento estaria mentindo ${ }^{(8,21)}$.

Diferentes métodos produzem dados nem sempre comparáveis; contudo, independente dos métodos utilizados nos diversos estudos disponíveis na literatura, fica evidente a ocorrência de adesão inadequada em proporção considerável de pacientes. Em doentes com DII a prevalência média de adesão, relatada nos diversos estudos, varia de $10 \%$ a $70 \%{ }^{(16,29,32)}$.

Entre os indivíduos com comportamento de nãoadesão é importante o esclarecimento dos motivos para tal atitude frente às recomendações de tratamento. A escala de Morisky e Green modificada, usada para Medida de Adesão ao Tratamento permitiu identificar que 57,8\% dos pacientes classificados como não-adesão apresentaram o comportamento não-intencional. Tal fato demonstra que estes tendem a negligenciar o tratamento por esquecimento ou descuido. Além disso, 39,1\% dos indivíduos classificados como não-adesão apresentam, simultaneamente, baixa disposição para o uso regular dos medicamentos prescritos. Tais pacientes apresentam interrupção voluntária do tratamento, comportamento este caracterizado como não-adesão do tipo intencional. Estes achados são similares a estudos prévios de não-adesão envolvendo doentes com DII ${ }^{(17,32)}$.

O método utilizado também permitiu investigar se a razão para o comportamento de não-adesão era possivelmente atribuída à falta de conhecimento (14\%), de motivação (22\%)ou ambas $(6,2 \%)$. Curiosamente, a maioria (37 pacientes; $57,8 \%$ ) que declararam não-adesão, apresentaram ter conhecimento e motivação para o tratamento.

Com relação aos possíveis determinantes de não-adesão, foi detectada uma possível associação entre não-adesão e idade mais jovem e raça não-branca. Isto pode estar relacionado, em parte, às questões de desigualdade vividas pela raça. Conforme o relatório do Programa das Nações Unidas para o Desenvolvimento ${ }^{(30)}$, independente da região do Brasil analisada e do grau de pobreza observado, os negros são sempre menos favorecidos com relação à inclusão no mercado de trabalho, acesso à educação, o que se reflete em pior condição social e, possivelmente, na dificuldade de aquisição de medicamentos que não são fornecidos gratuitamente. $\mathrm{Na}$ presente casuística, um número relativamente limitado de pacientes não-brancos foi incluído $(n=14)$. È possível admitir que, se a população negra participante do estudo fosse maior, a diferença estatística observada $(P=0.06)$ fosse mais significante. Entretanto, é bem conhecido que a DC é mais comum na raça branca, embora com a urbanização e assimilação do modo de vida ocidental, a frequência da DC em não-brancos venha aumentando ${ }^{(10)}$. Embora no estudo de EDIGER et al. ${ }^{\left({ }^{9}\right)}$ não fosse demonstrada correlação entre a raça e o grau de adesão, outros autores também verificaram que idade mais jovem correlaciona-se com não-adesão ${ }^{(5,9,25)}$. Indivíduos jovens, em geral, tendem a contrariar as regras, comportamento próprio da idade; possivelmente, a nãoadesão pode estar também associada ao fato do diagnóstico ser recente e à falta de vivência com relação ao curso clínico (atividade/remissão) da doença ${ }^{(5)}$.

$\mathrm{Na}$ presente série, as características clínicas da doença não demonstraram correlação significante com a não-adesão. Enquanto alguns autores avaliando pacientes com DII relataram associação entre não-adesão e atividade da doença ${ }^{(32)}$, outros reportaram correlação inversa ${ }^{(17,18,33)}$. Adicionalmente, na presente casuística, não foi observada associação entre a presença de depressão ou ansiedade e não-adesão. Entretanto, alguns estudos sugeriram que a adesão parece ser menor na vigência de estados emocionais negativos, como nos transtornos depressivos ou estresse emocional ${ }^{(21,29)}$. Por outro lado, estudo prévio identificou menor risco de não-adesão em pacientes com estresse ${ }^{(32)}$. É possível que pacientes sob estresse psíquico acreditem ter estado de saúde pior do que realmente tem e, desta forma, tendem a aderir mais rigorosamente ao tratamento prescrito. Do contrário, pacientes sem estresse psíquico parecem acreditar que o medicamento é desnecessário e tendem a apresentar menor grau de adesão ${ }^{(32)}$.

É importante ressaltar que o número algo limitado de pacientes alocados em algumas variáveis analisadas (tais como, tabagismo ou etilismo corrente), não possibilite a obtenção de evidências seguras do possível grau de influência destes hábitos no comportamento de não-adesão.

No presente estudo, a baixa taxa de tabagismo e alcoolismo pode ser justificada pelo acompanhamento sistemático dos pacientes nas consultas periódicas em ambulatórios, ocasião em que os mesmos recebem orientação sobre os possíveis efeitos deletérios do uso do tabaco e álcool sobre a DC e demais sistemas orgânicos.

O uso diário de medicamentos e o tipo de regime terapêutico (número de drogas e doses) têm sido apontados como fatores potencialmente associados ao grau de adesão em diversas doenças crônicas ${ }^{(15)}$. Grande número de medicamentos prescritos e esquema terapêutico complexo também estão associados à não-adesão, mesmo que o medicamento seja fornecido gratuitamente $^{(9,21)}$. Não obstante, neste estudo, o acesso gratuito aos medicamentos e o número de drogas utilizadas não se associaram ao grau de adesão apresentado pelo paciente. É admissível que o comportamento de não-adesão possa estar ligado a fatores subjetivos não contemplados neste estudo. Recomenda-se que novas abordagens sejam realizadas com o foco na compreensão do paciente quanto ao seu estado e na relação do paciente com o prescritor e a equipe multidisciplinar.

Destaca-se que os pacientes avaliados neste estudo eram assistidos em um centro secundário/terciário especializado em DII por diversos profissionais distintos. Assim, os achados desta série podem não refletir o grau de adesão de indivíduos com DC atendidos em ambientes de assistência primária ou privada, os quais possam ser assistidos periodicamente pelo mesmo profissional. Sabe-se que a relação médico-paciente estreita e estabelecida na base da confiança mútua, pode aumentar o grau de adesão ao tratamento ${ }^{(7,32)}$. 
Finalmente, estudos prospectivos longitudinais são necessários para verificar o impacto da não-adesão ao tratamento sobre a evolução clínica da DC e a possível utilidade das intervenções que visam melhorar o grau de adesão do paciente ao tratamento sobre o curso clínico desta afecção.

\section{CONCLUSÕES}

Neste estudo, dois terços dos pacientes com DC apresentaram não-adesão ao tratamento medicamentoso, predominando o comportamento não-intencional. O comportamento de não-adesão predominou em pacientes mais jovens e nãobrancos. É importante que se identifique, a priori, no início do tratamento, os pacientes com tendência a não-adesão, pois este comportamento reflete uma atitude geral do paciente com relação a sua saúde como um todo, não se restringindo à DC. Torna-se necessário que os profissionais e serviços de saúde assumam a corresponsabilidade nesse processo e que novos estudos sejam desenvolvidos para implementação de estratégias para minimizar o problema da não-adesão ao tratamento.

Cornélio RCAC, Pinto ALT, Pace FHL, Moraes JP, Chebli JMF. Non-adherence to the therapy in Crohn's disease patients: prevalence and risk factors. Arq Gastroenterol. 2009;46(3):183-9.

ABSTRACT - Context - Non-adherence to therapy, in any degree is a common event and occurs in several circumstances. It is one of most common cause of fail in therapy of chronic diseases and Crohn's disease is not an exception. Objective - To evaluate in patients with Crohn's disease the prevalence and the risk factors to non-adherence to therapy. Methods - From July 2006, for 12 months, were included prospectively, for non-adhesion to therapy 100 patients with Crohn's disease that were assisted in a Center for Inflammatory Bowel Diseases of Universitary Hospital of Federal University of Juiz de Fora, MG, in Brazil. A modified Morisky \& Green Test for Measure of Adherence to Therapy was answered by all of them. According to test the patients were classified in two groups defined as adherence and non-adherence, respectively, and the last one was separated in intentional and non-intentional adhesion. Clinical, psychological and pharmacotherapeutics variables were sought to find the factors related to non-adherence. Results - Sixty four percent of total group were noticed to have a score of non-adherence to therapy according to used test and non-intentional was the most common type of behavior in such patients, and they demonstrated to be conscious of therapy. The comparison of adherent and non-adherent patients displayed a significant tendency to occurrence of non-adherence in younger $(P=0,07)$ and in non-white patients $(P=0,06)$. No correlation was observed in comparison of psychological and pharmacotherapeutics variables and non-adherence. Conclusions - In patients with Crohn's disease the prevalence of non-adherence to therapy is high (64\%). The younger and non-white patients have higher propensity to non-adherence. In such circumstances efforts should be made to look for strategies to deal with this sort of people suffering from Crohn's disease, trying to increase the degree of adherence in this sort of patients.

HEADINGS - Crohn disease. Treatment refusal. Drug administration schedule.

\section{REFERÊNCIAS}

1. Beck AT, Ward CH, Mendelson M, Mock J, Erbaugh J. An inventory for measuring depression. Arch Gen Psychiatry. 1961;4:561-71.

2. Best WR, Becktel JM, Singleton JW, Kern F. Development of a Crohns disease activity index. National Cooperative Crohns Disease Study. Gastroenterology. 1976;70:439-44.

3. Botega NJ, Bio MR, Zomignani A, Garcia Jr C, Pereira WAB. Transtornos do humor em enfermaria de clínica médica e validação de escala de medida (HAD) de ansiedade e depressão. Rev Saúde Publica. 1995;29:355-63.

4. Case Management Society of America. Guidelines from the Case Management Society of America for improving patient adherence to medication therapies. CMGA-1, 2004.

5. D'Inca R, Bertomoro P, Mazzocco K, Vettorato MG, Rumiati R, Sturniolo GC. Risk factors for non-adherence to medication in inflammatory bowel disease. Aliment Pharmacol Ther. 2008;27:166-72.

6. Delgado AB, Lima ML. Contributo para a validação concorrente de uma medida de adesão aos tratamentos. Psicol Saúde Doenças. 2001;2:81-100.

7. Dewulf NL, Monteiro RA, Passos ADC, Troncon LE. Adesão ao tratamento medicamentoso em pacientes com doenças gastrointestinais crônicas acompanhados no ambulatório de um hospital universitário. Rev Bras Cienc Farm. 2006;42:575-84.

8. Dewulf NL, Monteiro RA, Passos ADC, Vieira EM, Troncon LE. Adesão ao tratamento medicamentoso de pacientes com doenças inflamatórias intestinais acompanhados no ambulatório de um hospital universitário. Arq Gastroenterol. 2007:44:289-96.

9. Ediger JP, Walker JR, Graff L, Lix L, Clara I, Rawsthorne P, Rogala L, Miller N, McPhail C, Deering K, Bernstein CN. Predictors of medication adherence in inflammatory bowel disease. Am J Gastroenterol. 2007;102:1417-26.

10. Fellows IW, Freeman JG, Holmes GK. Crohn's disease in the city of Derby, 1951-85. Gut. 1990;31:1262-5.

11. Gaburri PD, Chebli JM, Castro LE, Ferreira JO, Lopes MH, Ribeiro AM, Alves RA, Froese EC, Oliveira KS, Gaburri AK, Gaburri D, Meirellers GS, Souza AF.
Epidemiologia, aspectos clínicos e evolutivos da doença de Crohn: um estudo de 60 casos. Arq Gastroenterol. 1998;35:240-6.

12. Gasche C, Scholmerich J, Brynskov J, D’Haens G, Hanauer SB, Irvine EJ, Jewell DP, Rachmilewitz D, Sachar DB, Sandborn WJ, Sutherland LR. A simple classification of Crohn's disease: report of the Working Party for the World Congresses of Gastroenterology, Vienna, 1998. Inflamm Bowel Dis. 2000;6:8-15.

13. Gorenstein C, Andrade L. Validation of a Portuguese version of the Beck Depression Inventory and the State-Trait Anxiety inventory in Brazilian subjects. Braz J Med Biol Res. 1996;29:453-7.

14. Haynes B, Taylor BW, Sackett DL. Compliance in healthcare. Baltimore, Maryland: Univertsity Press; 1981.

15. Ickovics RJ, Meisler WA. Adherence in AIDS clinical trials: a framework for clinical research and care. J Clin Epidemiol. 1997;50:385-91.

16. Kane SV, Cohen RD, Aikens JE, Hanauer SB. Prevalence of nonadherence with maintenance mesalasine in quiescent ulcerative colitis. Am J Gastroenterol. 2001;96:2929-33.

17. Kane SV, Huo D, Aikens J, Hanauer S. Medication nonadherence and the outcomes of patients with quiescent ulcerative colitis. Am J Med. 2003;114:39-43.

18. Kane SV. Systematic review: adherence issues in the treatment of ulcerative colitis. Aliment Pharmacol Ther. 2006;23:577-85.

19. Kornbluth A, Sachar DB. Ulcerative colitis practice guidelines in adults (update): American College of Gastroenterology, Practice Parameters Committee. Am J Gastroenterol. 2004;99:1371-85.

20. Lashner BA. Clinical features, laboratory findings, and course of Crohn's disease. In: Kirsner JB, editor. Inflammatory bowel disease. Chicago: Saunders; 2000. p.305-14.

21. Leite SN, Vasconcellos MPC. Adesão à terapêutica medicamentosa: elementos para a discussão de conceitos e pressupostos adotados na literatura. Ciênc Saúde Coletiva. 2003;8:775-82

22. Lennard-Jones E. Classification of inflammatory bowel disease. Scand J Gastroenterol. 1989;24:2-6.

23. López San Román A, Bermejo F, Carrera E, Pérez-Abad M, Boixeda D. Adherence to treatment in inflammatory bowel disease. Rev Esp Enferm Dig. 2005;97:249-57. 
24. Mackner LM, Crandall WV. Oral medication adherence in pediatric inflammatory bowel disease. Inflamm Bowel Dis. 2005;11:1006-12.

25. Michaud PA, Suris JC, Viner R. The adolescent with a chronic condition. Part II: Healthcare provision. Arch Dis Child. 2004;89:943-9.

26. Milstein-Moscati I, Persano S, Castro LLC. Aspectos metodológicos e comportamentais da adesão à terapêutica. In: Castro LLC, org. Fundamentos de farmacoepidemiologia. São Paulo: Agrupuram; 2000. p.171-9.

27. Miszputen JS. Doenças inflamatórias intestinais - opções terapêuticas. J Bras Gastroenterol. 2007;7:5-60.

28. Morisky DE, Green W, Levine DM. Concurrent and predicitive validity of a self-reported measure of medication adherence. Med Care. 1986;24:67-74.

29. Nigro G, Angelini G, Grosso SB, Caula G, Sategna-Guidette C. Psychatric predictors of non compliance in inflammatory bowel disease. J Clin Gastroenterol. 2001;32:61-8.

30. Programa das Nações Unidas para o Desenvolvimento. [online]. 2008. Disponível em: http://www.pnud.org.br/raca/reportagens/index.php?id01=1608\&lay=rac.

31. Rose JDR, Roberts GM, Williams G, Mayberry JF, Rhodes J. Cardiff Crohn's disease jubilee: the incidence over 50 years. Gut. 1988;29:346-51.

32. Sewitch MJ, Abrahamowicz M, Barkun A, Bitton A, Wild GE, Cohen A, Dobkin PL. Patient nonadherence to medication in inflammatory bowel disease. Am J
Gastroenterol. 2003;98:1535-44.

33. Shale MJ, Riley AS. Studies of compliance with delayed-release mesalazine therapy in patients with inflammatory bowel disease. Aliment Pharmacol Ther. 2003;18:191-8.

34. Shanahan F. Crohn's disease. Lancet. 2002;359:62-9.

35. Souza MH, Troncon LE, Rodrigues CM, Viana CF, Onofre PH, Monteiro RA, Passos AD, Martinelli AL, Meneghelli UG. Evolução da ocorrência (19801999) da doença de Crohn e da retocolite ulcerativa idiopática e análise das suas características clínicas em um hospital universitário do sudeste do Brasil. Arq Gastroenterol. 2002;39:98-105.

36. Vlasnik JJ, Aliotta SL, DeLor B. Evidence-based assessment and intervention strategies to increase adherence to prescribed medication plans. Case Manager. 2005; 16:55-9.

37. Vlasnik JJ, Aliotta SL, DeLor B. Medication adherence: factors influencing complience with precribed medication plans. Case Manager. 2005;16:47-51.

38. Zigmond AS, Snaith RP. The hospital anxiety and depression scale. Acta Psychiatr Scand. 1983;67:361-70.

Recebido em 6/6/2008 Aprovado em 16/2/2009. 